\title{
MicroReview
}

\section{Evolution of fungal sex chromosomes}

\author{
James A. Fraser and Joseph Heitman* \\ Departments of Molecular Genetics and Microbiology, \\ Medicine, Pharmacology and Cancer Biology, Howard \\ Hughes Medical Institute, Duke University Medical Center, \\ Durham, NC 27710, USA.
}

\section{Summary}

Sexual reproduction enables organisms to shuffle two parental genomes to produce recombinant progeny, and to purge the genome of deleterious mutations. Sex is conserved in virtually all organisms, from bacteria and fungi to plants and animals, and yet the mechanisms by which sexual identity are established share both conserved general features and are remarkably diverse. In animals, sexual identity is established by dimorphic sex chromosomes, whereas in fungi a specialized region of the genome, known as the mating-type locus, governs the establishment of cell type identity and differs in DNA sequence between cells of different mating-types. Recent studies on the mating-type loci of fungi and algae reveal features shared with the mammalian $X$ and $Y$ chromosomes, suggesting that these represent early steps in the evolution of sex chromosomes.

Three general paradigms have dominated our understanding of eukaryotic biological systems over the past century. The first is that all organisms are composed of cells that are clonally derived from one another by mitotic cell divisions. The second is that chromosomes bear the genetic information and are responsible for inheritance. The third is that organisms reassort their genomes by sexual recombination, involving the fusion of mating partners or gametes that give rise to zygotes or diploid cells that undergo meiosis to produce progeny that may be better fitted to different environments.

Although there continues to be much debate as to the precise benefits of sexual recombination, it is clear that most organisms invest considerable energy and resources in this process. Two general models have

Accepted 9 October, 2003. *For correspondence. E-mail heitm001@ duke.edu; Tel. (+1) 919684 2824; Fax (+1) 9196845458. emerged: in one, sex allows the production of fitter recombinant progeny, and in the other it allows organisms to purge their genomes of deleterious mutations, such as errant transposable elements (Barton and Charlesworth, 1998; Lenski, 2001). In either case, the molecular mechanisms that enable organisms to differentiate into two distinct mating-types or sexes, and the processes of sexual reproduction via gamete production, cell fusion and meiosis are of considerable interest.

A striking difference between unicellular and multicellular eukaryotic organisms is that mating partners in fungi appear morphologically quite similar, in contrast to the sexual dimorphism that is characteristic of more complex systems. In fungi, cell identity is established by the mating-type locus. In the budding yeast Saccharomyces cerevisiae, cells that inherit the MATa allele of the matingtype locus are a-cells, and those that inherit the MAT $\alpha$ allele are $\alpha$-cells. Mating between a- and $\alpha$-cells results first in cell and then nuclear fusion to produce an $\mathbf{a} / \alpha$ diploid that can undergo meiosis (Herskowitz et al., 1992). The alleles of the MAT locus encode one or two key cell fate determinants, $\mathbf{a} 1$ (encoded by MATa), and $\alpha 1$ and $\alpha 2$ (encoded by $M A T \alpha$ ), which are DNA binding proteins that govern in trans expression of sex determining genes on other chromosomes (Herskowitz et al., 1992). The unique region of the mating-type locus in budding yeast is quite restricted and spans only $642 \mathrm{bp}$ in a-cells and $747 \mathrm{bp}$ in $\alpha$-cells, a small fraction of the $316613 \mathrm{bp}$ chromosome on which the MAT locus resides.

By contrast, in multicellular eukaryotes such as mice and humans, specialized sex chromosomes have evolved that govern differentiation and gamete production whereby $X X$ individuals are female and $X Y$ individuals are male. In this case, the $Y$ chromosome spans $\sim 50$ million $\mathrm{bp}$, many genes involved in sex determination and identity are located on the $X$ and $Y$ chromosomes, and the two sex chromosomes share only a restricted region that allows them to pair for proper segregation during meiosis (Skaletsky et al., 2003). The dramatically dimorphic sex chromosomes are thought to have evolved from an ancestral pair of autosomes by a series of steps. These are proposed to involve the conscription of the Sox3 factor to form the testis determining gene $S R Y$, a series of inversions that suppress recombination, expansion of the 
pseudoautosomal region shared between the incipient $X$ and $Y$ chromosomes, and then a series of duplications and gene conversion events that shaped the structures of the present day $X$ and $Y$ sex chromosomes (Charlesworth, 1991; Foster and Graves, 1994; Lahn and Page, 1999).

Although the mating-type loci of fungi and the sex chromosomes of mammals therefore appear quite divergent, recent studies of several fungal mating-type loci have revealed features shared with the more complex sex chromosomes and sex determining regions of algae, plants and animals (Fig. 1). We consider here three specific examples that highlight unique features of fungal MAT loci and mating-type chromosomes that provide insight into the evolution of sex chromosomes. First, in the plant fungal pathogen Ustilago hordei the two regions associated with tetrapolar mating-type systems are linked at the ends of a large complex region spanning $\sim 500000$ bp in which recombination is suppressed (Bakkeren and Kronstad, 1994; Lee et al., 1999). Second, in the filamentous fungus Neurospora tetrasperma the mating-type locus is located on an unusual chromosome within a large genomic region that fails to pair during meiosis, and this mating-type specific region is flanked by a common region in which an obligate recombination event occurs (Merino et al., 1996; Gallegos et al., 2000) and third, in the human fungal pathogen Cryptococcus neoformans the mating-type locus spans $>100000 \mathrm{bp}$, encodes $\sim 20$ distinct genes, and is composed almost entirely of divergent alleles of a common gene set (Moore and Edman, 1993; Karos et al., 2000; Lengeler et al., 2002). In comparison with these fungal mating systems, we also point out unusual aspects of sex determination in Pneumocystis carinii, Microbotryum violaceum and other representative fungi. Finally, we will consider these fungal sex-determining mechanisms in comparison to a similar region of the Chlamydomonas reinhardtii genome that defines the algal mating-type locus that also shares features with sex chromosomes (Ferris and Goodenough, 1994; Ferris et al., 2002).

Ustilago hordei is a basidiomycetous fungus that infects barley and is evolutionarily related to Ustilago maydis, which infects maize plants. Ustilago maydis has a tetrapolar system with many mating-types, whereas $U$. hordei has a bipolar system with only two mating-types. In $U$. maydis, the a mating-type locus encodes pheromones and pheromone receptors involved in mate recognition and cell-cell fusion during mating (Bölker et al., 1992). The $b$ mating-type locus is on a different chromosome and encodes divergent homeodomain proteins, bE and bW, that heterodimerize to establish cell identity following cell fusion (Kronstad and Leong, 1989; 1990; Kämper et al., 1995; Kahmann and Bölker, 1996; Kronstad and Staben, 1997). The $b$ locus is multiallelic, giving rise to dozens of distinct mating-types that promote outbreeding. Because the $a$ and $b$ loci are unlinked, and mating only occurs when both differ, $U$. maydis has a tetrapolar mating system in which any given meiotic segregant can only mate with $25 \%$ of the progeny of the cross ( $a 1 b 1$ mates with $a 2$ b2 but not with $a 1 b 1, a 1 b 2$, or a2 b1). This tetrapolar mating system has been expanded in the wood-rotting fungus Schizophyllum commune and the model mushroom Coprinus cinereus, in which the $a$ and $b$ MAT loci are tandemly duplicated or triplicated and multiallelic, giving rise to thousands of mating-types (Kües et al., 1992; Specht et al., 1992; Stankis et al., 1992; Wendland et al., 1995; Casselton and Olesnicky, 1998; Casselton, 2002).

Elegant molecular studies conducted by James Kronstad and colleagues revealed that the $a$ and $b$ mating-type loci are linked in U. hordei (Bakkeren and Kronstad, 1994; Lee et al., 1999). Thus, the mating-type loci that are known to span only $\sim 4 \mathrm{~kb}$ (b locus) and $\sim 4.5-8 \mathrm{~kb}$ ( $a$ locus) in the tetrapolar system of $U$. maydis are dramatically expanded in U. hordei to cover now $\sim 500000 \mathrm{bp}$, or about one-sixth of the $\sim 2.8$ million bp chromosome on which the MAT locus now resides. This expansion of the sex-determining region in a bipolar system to occupy a substantial portion of a chromosome is a key feature shared with the mammalian sex chromosomes. In addition, the $U$. hordei mating-type locus is no longer multiallelic, resulting in only two different mating-type alleles, MAT-1 (a1 b1, separated by $500 \mathrm{~kb}$ ) and MAT-2 (a2 b2, separated by $430 \mathrm{~kb}$ ), to give rise to a bipolar system.

The second key feature that the $U$. hordei MAT locus shares with more complex sex chromosomes is that recombination is suppressed across the entire region between the $a$ and $b$ loci at the distal ends of the MAT locus. Based on sequence analysis of BAC clones spanning this region, the mechanisms that give rise to this recombinational suppression likely involve chromosomal rearrangements and the presence of many transposable and repetitive elements within the $\sim 430$ 000-500 000 bp intervening region (J. Kronstad, pers. comm.). Whether additional genes involved in mating-type determination have been incorporated into the locus between the $a$ and $b$ mating-type loci, or lie outside but linked to the MAT locus is not known. It is striking that heterologous expression studies have revealed that the $U$. hordei $b$ genes are functional in $U$. maydis, further illustrating the close evolutionary link between the two fungi (Bakkeren and Kronstad, 1993). Whether an ancestral tetrapolar system, such as in $U$. maydis, gave rise to this bipolar system by fusion and linkage, or whether a bipolar system, such as in $U$. hordei, gave rise to the tetrapolar system by chromosomal translocation and allele divergence is a matter of much debate (Raper, 1966). In our view, it is likely that the bipolar system was the ancestral one, given that it occurs in both ascomycetes and basidiomycetes. Evolution likely then occurred from bipolar to tetrapolar and 


\section{U. maydis}
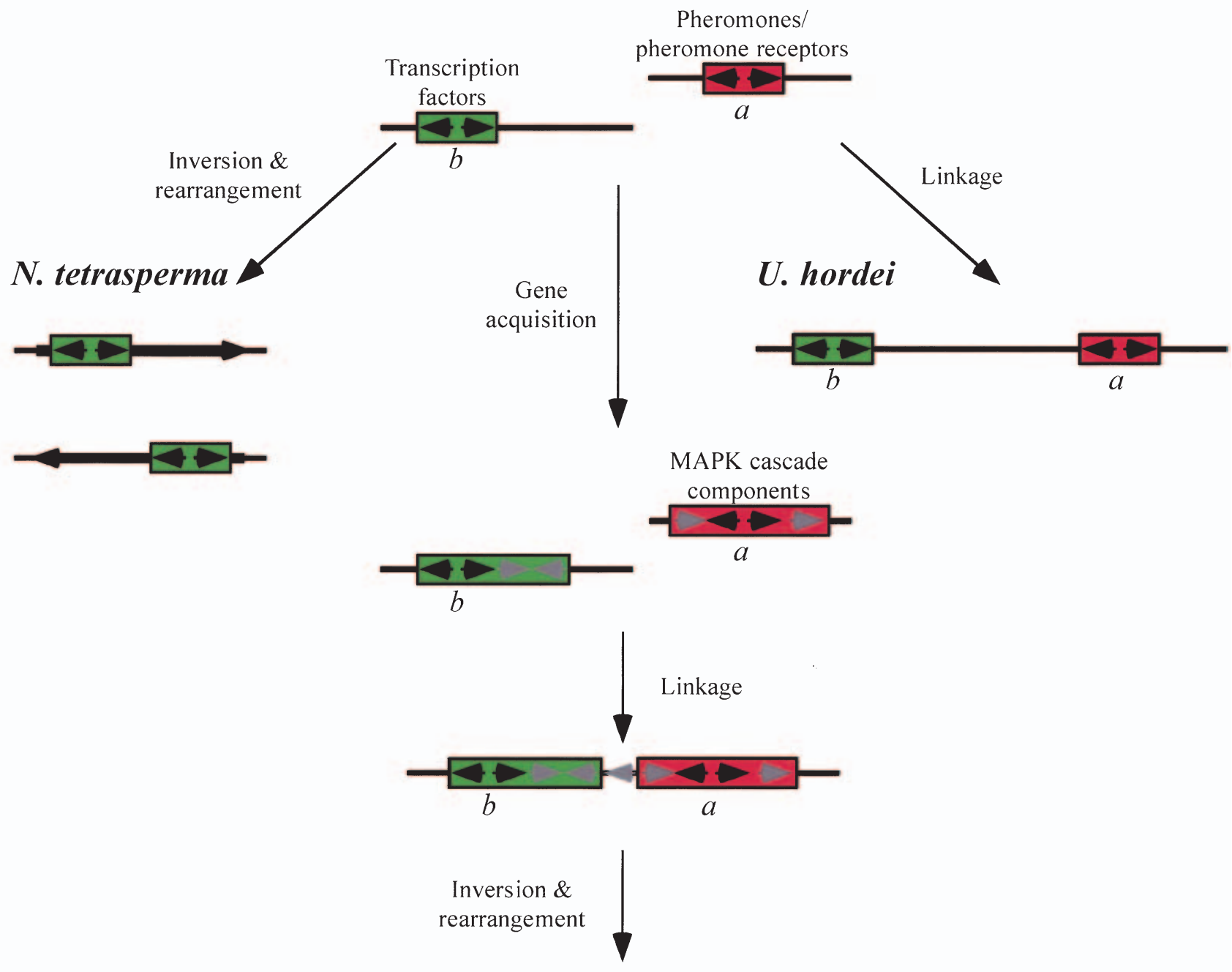

\section{C. neoformans}

Fig. 1. Evolution of fungal mating-type loci shares features with the evolution of sex chromosomes. Fungi have either bipolar or tetrapolar matingtype systems that govern the interaction of partners during the sexual cycle. Fungi with bipolar systems exist as two alternative mating-types whose cell fates are governed by a single chromosomal locus with two alleles that encode homeodomain, HMG box, or other classes of transcription factors. The situation is more complex in fungi with tetrapolar mating-type systems, in which two different chromosomal loci govern cell-cell interactions during mating, with allelic differences required at both loci for completion of the sexual cycle. In these systems, one locus ( $b$ in U. maydis) encodes homeodomain transcription factors, and the other ( $a$ in U. maydis) encodes pheromones and pheromone receptors. In addition, in many fungi with tetrapolar mating-type systems, including C. cinereus and S. commune, the MAT loci are multiallelic, giving rise to hundreds to thousands of different mating-types. The evolution and function of these unique genomic loci serve as valuable models for our understanding of complex cell-cell recognition events and the establishment of cell identity. Several fungal MAT loci also share features with the more complex sex chromosomes of multicellular eukaryotes. In $N$. tetrasperma the size restricted bipolar mating-type locus typical of other ascomycetous fungi appears to have been conscripted by a large chromosomal inversion and rearrangement, such that the MAT locus now occupies much of the chromosome on which it resides. In U. hordei, the $a$ and $b$ MAT loci that correspond to the tetrapolar MAT loci of U. maydis are linked, resulting in a bipolar system in which recombination is now suppressed over the large intervening region between the two loci. A more complex series of events has transpired during the evolution of the mating-type locus of the pathogenic basidiomycete $C$. neoformans. First, additional genes have been acquired and linked to the ancestral $a$ and $b M A T$ loci, including several additional elements of the pheromone activated MAP kinase cascade. Second, the ancestral $a$ and $b$ MAT loci have been linked into one common contiguous chromosomal region, possibly entrapping unrelated genes between the two regions. Finally, additional inversions and rearrangements have occurred, giving rise to the current structure of the mating-type locus that has been defined in two divergent varieties of the organism. The evolutionary steps that gave rise to these unique fungal mating-type loci are analogous in several respects with the events hypothesized during the evolution of sex chromosomes in multicellular eukaryotes. 
perhaps back to bipolar in some specific examples (e.g. U. hordei).

Like U. maydis, Cryptococcus neoformans is a pathogenic basidiomycetous fungus in which mating-type has been linked to virulence (Hull and Heitman, 2002). Whereas for $U$. maydis it is the dikaryon that grows filamentously, infects maize plants, and completes the sexual cycle in planta, for $C$. neoformans it is the yeast form that infects humans, and mating and filamentation are not known to occur in the host. Also, $C$. neoformans is unique in that the $\alpha$-mating type is more common in the environment and can be more infectious in animal models (KwonChung et al., 1992). These findings have focused interest on the structure and function of the mating-type locus.

Similar to $U$. hordei, $C$. neoformans has a bipolar mating-type system involving fusion between haploid a- and $\alpha$-cells. The a and $\alpha$ alleles of the mating-type locus from isolates of two divergent varieties of $C$. neoformans have been cloned and sequenced (Lengeler et al., 2002). The MAT locus spans $>100 \mathrm{~kb}$ and contains more than 20 genes, some of which function in differentiation and virulence and others that have no clear link to either. The one $\alpha$-unique gene encodes a novel HD1 class homeodomain factor, Sxi1 $\alpha$, which functions as a key cell fate determinant to establish $\mathbf{a} / \alpha$-cell identity (Hull et al., 2002). Remarkably, the a and $\alpha$ alleles are otherwise composed of divergent sets of the same cohort of genes, and therefore evolved from a common ancestral DNA region that has been extensively remodelled. Most interestingly, these genes include not only pheromones and pheromone receptors, as in other basidiomycetes, but also several of the elements of the pheromone responsive MAP kinase cascade itself. Recombination is suppressed across the entire region, likely the result of inversions and rearrangements, gene divergence, and the presence of many transposons, transposon remnants, and repetitive sequences through which the locus may expand or contract. This locus is reminiscent of the mating-type locus of $U$. hordei in that genes contained within the $a$ and $b$ mating-type loci of other basidiomycetes are present (e.g. those encoding pheromones, pheromone receptors, and homeodomain proteins), but in $C$. neoformans they are now also combined with many novel genes and have been condensed into one contiguous region of the genome. These studies establish a novel paradigm for the arrangement of fungal mating-type information that shares features with both known fungal MAT loci and the larger, more complex sex chromosomes of animals and plants.

Neurospora tetrasperma is closely related to the model filamentous ascomycete Neurospora crassa and yet its mating-type locus has undergone a dramatic rearrangement and now occupies a substantial portion of the host chromosome (Gallegos et al., 2000). The mating-type locus of $N$. crassa has been well characterized, spans a relatively small region of the genome (5301 bp for mat $A$ and 3235 bp for mat a), encodes one to three DNA binding proteins that establish cell identity, and shows both linkage and recombination with other regions of the host chromosome (Kronstad and Staben, 1997). By contrast, the mating-type locus of $N$. tetrasperma resides in a related chromosome in which the vast majority is recombinationally suppressed, and allelic differences are common in genes derived from the a- and A-mating type determining regions (Merino et al., 1996; Gallegos et al., 2000). Cytological analysis shows that a very large region of the mating-type chromosome fails to pair during meiosis, and this is correlated with the region in which recombination is suppressed. Most interestingly, there is also an obligate recombination event that occurs on the left end of the chromosome and which may function to ensure proper segregation, analogous to the pseudoautosomal region of the mammalian $X$ and $Y$ sex chromosomes. The mechanisms responsible for recombinational suppression or activation of the $N$. tetrasperma mating-type chromosomes are not yet understood but may involve both inversions, rearrangements and sequence divergence to suppress recombination, and DNA lesion generating systems (breaks, nicks or DNA bound proteins) to stimulate recombination and ensure faithful chromosome disjunction at meiosis.

Two other fungi that contain unusual genomic regions involved or implicated in sex determination are Pneumocystis carinii and Microbotryum violaceum. In the ascomycetous human fungal pathogen $P$. carinii, a novel region of the genome bears a striking resemblance to the matingtype locus of $C$. neoformans (Smulian et al., 2001). This region contains genes encoding homologues of a pheromone and pheromone receptor, and also several elements of a MAP kinase cascade including homologues of the Ste20 PAK kinase and the Ste12 transcription factor. Whereas $P$. carinii has no known sexual cycle and cannot be cultured outside of the host, pathological analysis of infected lung tissue has revealed morphological features that suggest sexual recombination and meiosis might be occurring during infection. Whether or not this unique region of the genome is involved in sex determination, or whether $P$. carinii even has a sexual cycle, remain to be established, but it is quite intriguing that divergent ascomycetous and basidiomycetous human fungal pathogens share a clustering of signalling elements known or predicted to be involved in mating, and thus this unique genomic arrangement may have predated the divergence of the two fungal phyla.

Microbotryum violaceum is a basidiomycetous fungus that causes anther smut disease of flowering plants. Mating of this phytopathogen is controlled by two mating-type alleles, a1 and a2, and the organism exhibits intratetrad mating and the presence of haploid lethal genes linked to 
the a2 mating-type. Most interestingly, recent studies reveal that the a1 and a2 mating-type chromosomes are markedly different in size, spanning 2.9 and 3.5 million bp respectively (Hood, 2002). Little is known in detail about the structure or extent of the mating-type specific sequences that reside on these size dimorphic fungal sex chromosomes, but there is emerging evidence that recombination is suppressed and that there is a marked abundance of transposable elements compared to the autosomes (M.E. Hood and S. Antonovics, pers. comm.).

These fungal sex chromosomes share several features that are reminiscent of a specialized region of the genome of the alga Chlamydomonas reinhardtii that establishes the two mating-types, mt- and $\mathrm{mt}+$, in this unicellular eukaryotic microorganism. Ursula Goodenough and colleagues have defined a unique region of the algal genome that spans some $830000 \mathrm{bp}$ and which contains a core region of $190000 \mathrm{bp}$ encoding several genes involved in agglutination of mating partners, cell fusion, and the establishment of cell identity (Ferris and Goodenough, 1994; Ferris et al., 2002). This includes the Mid gene, which is unique to the mt- locus and encodes a candidate transcriptional regulator that controls differentiation of $\mathrm{mt}-$ gametes. mt+ gametes express the Gsp1 homeodomain factor, and heterologous misexpression of Gsp1 induces mt- gametes to form zygotes; interestingly, Gsp1 is not encoded by the mating-type locus but must be under control of an mt+ specific factor (Zhao et al., 2001). Thus, the algal cell identity system shares features with the fungal homeodomain factors. The core $190000 \mathrm{bp}$ region of the algal MAT locus contains extensive alterations between the $\mathrm{mt}+$ and $\mathrm{mt}$ - alleles, including inversions, translocations, gene duplications and mutations. The locus also contains many transposable elements and remnants and repetitive sequences. These structural aberrations result in suppression of recombination over the core and flanking sequences that span almost one million basepairs. Thus, the algal MAT locus shares several features with both fungal MAT loci and the more complex sex chromosomes of mammals.

Studies in Chlamydomonas also reveal another unique feature associated with fungal, algal, and mammalian sex determining regions involving uniparental inheritance of cytoplasmic organelles. In Chlamydomonas, mating results in the uniparental inheritance of chloroplasts from the $\mathrm{mt}+$ parent (Armbrust et al., 1993) through mechanisms that are only now coming to be understood (Umen and Goodenough, 2001; Nishimura et al., 2002). Uniparental inheritance of mitochondria occurs in diverse fungi, including N. tetrasperma, Aspergillus nidulans, and others (Lee and Taylor, 1993; Coenen et al., 1996). Recent studies demonstrate that mitochondria are also uniparentally inherited in $C$. neoformans, in this case from the a matingtype parent following mating (Yan and $\mathrm{Xu}, 2003$ ). These findings are strikingly similar to the uniparental maternal inheritance of mitochondria that occurs in mammals. Uniparental inheritance is thought to restrict organelle transfer to the zygote and thereby inhibit selfish DNA elements from commandeering the organellar gene pool.

This comparison of divergent sex chromosomes suggests that it should be possible to retrace the evolutionary history of fungal/algal sex chromosomes, analogous to recent hypotheses on the evolution of the human $X$ and $Y$ sex chromosomes (Lahn and Page, 1999). As described earlier, mammalian sex chromosomes are thought to have evolved from an ancestral pair of autosomes that first acquired a sex determining gene, and then diverged via a series of inversions and rearrangments, becoming recombinationally isolated from each other except within the pseudoautosomal region. In a diploid organism, this can then lead to degeneration of one of the sex chromosomes and the evolution of dosage compensation mechanisms. Comparison to the fungal mating-type loci suggests similar molecular mechanisms may also have operated in this kingdom.

We consider first the evolution of mating-types and the MAT locus and then the expansion of these loci to fungal sex chromosomes. In ascomycetous and basidiomycetous fungi in which it is known, the mating-type locus encodes transcriptional control elements, including proteins with homeodomains, $\alpha$-domains, HMG boxes, or other novel regulatory motifs. Perhaps the ancestral two mating-types were established by epigenetic silencing of one common region, such that cells expressing the locus were one mating-type and cells not expressing the locus were of the opposite mating-type. An analogy is that in $S$. cerevisiae, cells in which the MAT locus has been deleted mate as a-cells, and are denoted a-like faker or 'alf' cells because they fuse with $\alpha$ cells but the resulting $\alpha / \Delta$ diploid cells fail to undergo meiosis (Herskowitz et al., 1992).

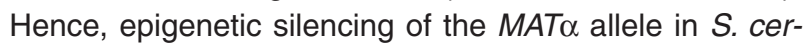
evisiae would yield cells of a mating-type competent to fuse with non-silenced $\alpha$ members of the population. Evolution of a distinct opposite mating-type allele could then have occurred in the next step, either by conscripting a transcriptional regulator unrelated to those of the opposite $M A T$ allele, or by duplication and sequence divergence of the original MAT allele into the distinct HD1 and HD2 classes to enable not only self-self homodimeric interactions but also self-non-self heterodimerization. This ancestral state may be reflected in the ability of the $S$. cerevisiae HD1 factor $\alpha 2$ to form both self HD1-HD1 homodimeric $(\alpha 2 / \alpha 2)$ and self-non-self HD1-HD2 heterodimeric $(\mathbf{a} 1 / \alpha 2)$ regulatory complexes. In either model, the association of key transcriptional regulators within the MAT locus clearly preceded the divergence of the ascomycetes and the basidiomycetes. In the basidiomycete lineage, an additional association of elements of the pher- 
omone production and sensing pathway with the matingtype locus has occurred.

An alternative model for the earliest steps in MAT locus evolution can be proposed based on the paired arrangement of linked genes involved in sexual identity in $U$. maydis and other basidiomycetes. In this model, an ancestral paired set of genes encoding interacting products (HD1 and HD2 homeodomain proteins, for example) could have directed a homothallic lifestyle involving selffusion. Next, gene mutation or loss occurred to yield heterothallic partners that express only one or the other cell identity determinant, and self-non-self cell fusion then became necessary to allow HD1-HD2 heterodimerization and completion of the sexual cycle. This model is sup-

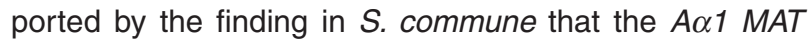
locus allele encodes only one HD2 factor, Y1, whereas the compatible $A \alpha 3$ allele encodes divergent HD1 and HD2 elements (Z3 and Y3), and only Y1-Z3 heterodimers form following cell fusion to drive sexual development (Specht

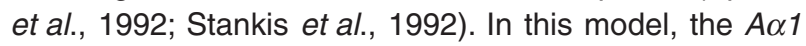
MAT locus allele has lost the gene encoding the ancestral paired HD1 factor. Such a mechanism could also have contributed to the evolution of the S. cerevisiae MAT locus. In this case, the MATa allele still encodes an active HD2 factor (a1), and also a non-functional HD1 factor remnant (a2), whereas the MAT $\alpha$ allele encodes an HD1 factor $(\alpha 2)$ but, in this model, the ancestral gene encoding an HD2 class factor has been replaced by one encoding the $\alpha$-domain protein $\alpha 1$ (Herskowitz et al., 1992). This model could also resolve the long-standing mystery of why the a2 gene contained within the MATa allele of the S. cerevisiae MAT locus encodes a conserved but truncated form of the $\alpha 2$ HD1 factor with no ascribed function.

Fungal sex chromosomes likely then evolved via several distinct mechanisms (Fig. 1). First, from studies in the bipolar fungus $U$. hordei it is clear that linkage of discrete genomic regions, possibly via chromosomal translocations, may have driven the clustering of sex related genes and subsequent inversions and rearrangements resulted in suppression of recombination. Second, the large mating-type chromosome of $N$. tetrasperma suggests that an initial chromosomal inversion event, possibly followed by subsequent rearrangements that block meiotic pairing, isolated a large genomic region rapidly given that $N$. crassa and $N$. tetrasperma are closely related in evolution.

In the human fungal pathogen $C$. neoformans, the evolution of a large contiguous mating-type locus in which many mating specific genes have become linked requires additional evolutionary steps (Fig. 1). First, we suggest that the genes encoding elements of the pheromone response pathway initially became linked on the same chromosome as the ancestral locus encoding pheromones and pheromone receptors. Second, these additional mating genes were incorporated into the MAT locus by a series of chromosomal inversions. Third, the two isolated regions encoding homeodomain proteins and the pheromone response pathway elements were linked by a chromosomal translocation in one mating-type. This translocation may have then selected for a compatible translocation event to result in linkage of the two loci in the opposite mating-type. The pairing of a translocated homologue with the original non-translocated homologues results in meiotic segregation in either of two patterns: adjacent-1 segregation to yield inviable products or alternate segregation to yield viable products. It is possible that during pairing and segregation of the translocated MAT locus chromosome with the non-translocated chromosomes harbouring the unlinked opposite mating-type allele that pairing, chromosomal breaks, and religation events promote similar, compatible translocation events. Finally, the region between the two now linked MAT loci may have contracted and then been subjected to a series of inversions and rearrangements to give rise to the present organization of the mating-type locus as one contiguous region that may be bounded by recombinational activators to prevent its spread to the entire chromosome. These models make the testable predictions that in other fungal genomes we may observe: (i) the MAT loci and genes encoding pheromone response pathway elements present on the same chromosome; (ii) linkage of pheromone response pathway genes and other homeodomain and HMG box proteins within the MAT locus, and (iii) other examples of linked MAT loci without contraction of the intervening sequences, similar to $U$. hordei. The incorporation of knowledge gained through multiple ongoing fungal genome sequencing projects (Whitehead Institute, Fungal Genome Initiative and others) will provide the means to test these hypotheses and provide further insights into mating locus structures and functions.

In closing, it is quite remarkable that the sex determining regions of fungi, algae, and animals share conserved structural features, and all the more remarkable that three key biological events are under the common control of this regulatory hierarchy in these evolutionarily divergent organisms. The mating-type loci and sex chromosomes have evolved to coordinately control: (i) gamete production and the formation of zygotes (ii) the establishment of the diploid or dikaryotic state, and (iii) the uniparental inheritance of organelles. Our challenge is to understand how these complex sex determining systems evolved, and to elucidate the molecular mechanisms by which they exert regulatory control to coordinate sexual recombination and enable evolutionary selection.

\section{Acknowledgements}

We thank Jim Kronstad and Blanche Capel for discussions and comments on the manuscript, Robert Marra for 
discussions and Lorna Casselton for stimulating and insightful comments on early events in MAT locus evolution. Our research studies are supported by grants from the NIH/NIAID, the Burroughs Wellcome Fund, and the Howard Hughes Medical Institute.

\section{References}

Armbrust, E.V., Ferris, P.J., and Goodenough, U.W. (1993) A mating type-linked gene cluster expressed in Chlamydomonas zygotes participates in the uniparental inheritance of the chloroplast genome. Cell 74: 801-811.

Bakkeren, G., and Kronstad, J.W. (1993) Conservation of the $b$ mating-type gene complex among bipolar and tetrapolar smut fungi. The Plant Cell 5: 123-136.

Bakkeren, G., and Kronstad, J.W. (1994) Linkage of matingtype loci distinguishes bipolar from tetrapolar mating in basidiomycetous smut fungi. Proc Natl Acad Sci USA 91: 7085-7089.

Barton, N.H., and Charlesworth, B. (1998) Why sex and recombination? Science 281: 1986-1990.

Bölker, M., Urban, M., and Kahmann, R. (1992) The a mating type locus of $U$. maydis specifies cell signaling components. Cell 68: 441-450.

Casselton, L.A. (2002) Mate recognition in fungi. Heredity $\mathbf{8 8}$ 142-147.

Casselton, L.A., and Olesnicky, N.S. (1998) Molecular genetics of mating recognition in basidiomycete fungi. Microbiol Mol Biol Rev 62: 55-70.

Charlesworth, B. (1991) The evolution of sex chromosomes. Science 251: 1030-1033.

Coenen, A., Croft, J.H., Slakhorst, M., Debets, F., and Hoekstra, R. (1996) Mitochondrial inheritance in Aspergillus nidulans. Genet Res 67: 93-100.

Ferris, P.J., and Goodenough, U.W. (1994) The mating-type locus of Chlamydomonas reinhardtii contains highly rearranged DNA sequences. Cell 76: 1135-1145.

Ferris, P.J., Armbrust, E.V., and Goodenough, U.W. (2002) Genetic structure of the mating-type locus of Chlamydomonas reinhardtii. Genetics 160: 181-200.

Foster, J.W., and Graves, J.A. (1994) An SRY-related sequence on the marsupial $X$ chromosome: implications for the evolution of the mammalian testis-determining gene. Proc Natl Acad Sci USA 91: 1927-1931.

Gallegos, A., Jacobson, D.J., Raju, N.B., Skupski, M.P., and Natvig, D.O. (2000) Suppressed recombination and a pairing anomaly on the mating-type chromosome of Neurospora tetrasperma. Genetics 154: 623-633.

Herskowitz, I., Rine, J., and Strathern, J.N. (1992) Matingtype determination and mating-type interconversion in Saccharomyces cerevisiae. In The Molecular and Cellular Biology of the Yeast Saccharomyces, Vol. 2. Jones, E.W., Pringle, J.R., Broach, J.R., (eds). Plainview: Cold Spring Harbor Laboratory Press, pp. 583-656.

Hood, M.E. (2002) Dimorphic mating-type chromosomes in the fungus Microbotryum violaceum. Genetics 160: 457461.

Hull, C.M., and Heitman, J. (2002) Genetics of Cryptococcus neoformans. Annu Rev Genet 36: 557-615.

Hull, C.M., Davidson, R.C., and Heitman, J. (2002) Cell iden- tity and sexual development in Cryptococcus neoformans are controlled by the mating-type-specific homeodomain protein Sxi1 $\alpha$. Genes Dev 16: 3046-3060.

Kahmann, R., and Bölker, M. (1996) Self/nonself recognition in fungi: old mysteries and simple solutions. Cell 85: 145148.

Kämper, J., Reichmann, M., Romeis, T., Bölker, M., and Kahmann, R. (1995) Multiallelic recognition: nonselfdependent dimerization of the $\mathrm{bE}$ and $\mathrm{bW}$ homeodomain proteins in Ustilago maydis. Cell 81: 73-83.

Karos, M., Chang, Y.C., McClelland, C.M., Clarke, D.L., Fu, J., Wickes, B.L., and Kwon-Chung, K.J. (2000) Mapping of the Cryptococcus neoformans MAT $\alpha$ locus: presence of mating type-specific mitogen-activated protein kinase cascade homologs. J Bacteriol 182: 6222-6227.

Kronstad, J.W., and Leong, S.A. (1989) Isolation of two alleles of the $b$ locus of Ustilago maydis. Proc Natl Acad Sci USA 86: 978-982.

Kronstad, J.W., and Leong, S.A. (1990) The $b$ mating-type locus of Ustilago maydis contains variable and constant regions. Genes Dev 4: 1384-1395.

Kronstad, J.W., and Staben, C. (1997) Mating type in filamentous fungi. Annu Rev Genet 31: 245-276.

Kües, U., Richardson, W.V., Tymon, A.M., Mutasa, E.S., Gottgens, B., Gaubatz, S., et al. (1992) The combination of dissimilar alleles of the A alpha and A beta gene complexes, whose proteins contain homeo domain motifs, determines sexual development in the mushroom Coprinus cinereus. Genes Dev 6: 568-577.

Kwon-Chung, K.J., Edman, J.C., and Wickes, B.L. (1992) Genetic association of mating types and virulence in Cryptococcus neoformans. Infect Immun 60: 602-605.

Lahn, B.T., and Page, D.C. (1999) Four evolutionary strata on the human X chromosome. Science 286: 964-967.

Lee, S.B., and Taylor, J.W. (1993) Uniparental inheritance and replacement of mitochondrial DNA in Neurospora tetrasperma. Genetics 134: 1063-1075.

Lee, N., Bakkeren, G., Wong, K., Sherwood, J.E., and Kronstad, J.W. (1999) The mating-type and pathogenicity locus of the fungus Ustilago hordei spans a $500-\mathrm{kb}$ region. Proc Natl Acad Sci USA 96: 15026-15031.

Lengeler, K.B., Fox, D.S., Fraser, J.A., Allen, A., Forrester, K., Dietrich, F.S., and Heitman, J. (2002) Mating-type locus of Cryptococcus neoformans: a step in the evolution of sex chromosomes. Eukaryot Cell 1: 704-718.

Lenski, R.E. (2001) Genetics and evolution. Come fly, and leave the baggage behind. Science 294: 533-534.

Merino, S.T., Nelson, M.A., Jacobson, D.J., and Natvig, D.O. (1996) Pseudohomothallism and evolution of the matingtype chromosome in Neurospora tetrasperma. Genetics 143: 789-799.

Moore, T.D.E., and Edman, J.C. (1993) The $\alpha$-mating type locus of Cryptococcus neoformans contains a peptide pheromone gene. Mol Cell Biol 13: 1962-1970.

Nishimura, Y., Misumi, O., Kato, K., Inada, N., Higashiyama, T., Momoyama, Y., and Kuroiwa, T. (2002) An mt (+) gamete-specific nuclease that targets $\mathrm{mt}(-)$ chloroplasts during sexual reproduction in C. reinhardtii. Genes Dev 16: 1116-1128.

Raper, J.R. (1966) Genetics of Sexuality in Higher Fungi. New York: Ronald Press Co. 
Skaletsky, H., Kuroda-Kawaguchi, T., Minx, P.J., Cordum, H.S., Hillier, L., Brown, L.G., et al. (2003) The male-specific region of the human $Y$ chromosome is a mosaic of discrete sequence classes. Nature 423: 825-837.

Smulian, A.G., Sesterhenn, T., Tanaka, R., and Cushion, M.T. (2001) The ste3 pheromone receptor gene of Pneumocystis carinii is surrounded by a cluster of signal transduction genes. Genetics 157: 991-1002.

Specht, C.A., Stankis, M.M., Giasson, L., Novotny, C.P., and Ullrich, R.C. (1992) Functional analysis of the homeodomain-related proteins of the A alpha locus of Schizophyllum commune. Proc Natl Acad Sci USA 89: 71747178.

Stankis, M.M., Specht, C.A., Yang, H., Giasson, L., Ullrich, R.C., and Novotny, C.P. (1992) The A alpha mating locus of Schizophyllum commune encodes two dissimilar multiallelic homeodomain proteins. Proc Natl Acad Sci USA 89: 7169-7173.
Umen, J.G., and Goodenough, U.W. (2001) Chloroplast DNA methylation and inheritance in Chlamydomonas. Genes Dev 15: 2585-2597.

Wendland, J., Waillancourt, L.J., Hegner, J., Lengeler, K.B., Laddison, K.J., Specht, C.A., et al. (1995) The mating-type locus B alpha 1 of Schizophyllum commune contains a pheromone receptor gene and putative pheromone genes. EMBO J 14: 5271-5278.

Yan, X., and Xu, J. (2003) Mitochondria are inherited from the MATa parent in crosses of the basidiomycete fungus Cryptococcus neoformans. Genetics 163: 13151325.

Zhao, H., Lu, M., Singh, R., and Snell, W.J. (2001) Ectopic expression of a Chlamydomonas $\mathrm{mt}+$-specific homeodomain protein in mt- gametes initiates zygote development without gamete fusion. Genes Dev 15: 2767-2777. 